\title{
A positive Liapunov exponent for the critical value of an S-unimodal mapping implies uniform hyperbolicity
}

\author{
TOMASZ NOWICKI \\ Warsaw Agricultural University, Department of Applied Mathematics, \\ 02-766 Warsaw 166 Nowoursynowska, Poland
}

(Received 3 November 1986; revised 3 June 1987)

\begin{abstract}
A positive Liapunov exponent for the critical value of an $S$-unimodal mapping implies a positive Liapunov exponent of the backward orbit of the critical point, uniform hyperbolic structure on the set of periodic points and an exponential diminution of the length of the intervals of monotonicity. This is the proof of the Collet-Eckmann conjecture from 1981 in the general case.
\end{abstract}

\section{Introduction}

The existence of an invariant measure absolutely continuous with respect to Lebesgue measure for maps on the interval is often connected with the existence of some hyperbolic structure i.e. the exponential growth of derivatives of the iterations on some subset of the interval. (But, according to $[\mathbf{B}, \mathbf{C}]$, sometimes there is only subexponential growth.)

By virtue of examples we recall the works of Lasota and Yorke [L, Y], Ruelle [R], Misiurewicz [M] and Szlenk [Sz] where the assumed conditions, sufficient for the existence of the absolutely continuous invariant measure, imply the uniform hyperbolic structure over the whole interval, the image of the critical point or some Cantor set.

In 1981 Collet and Eckmann [CE1] proved the existence of such a measure for $S$-unimodal mappings which have the uniform hyperbolic structure on the image of critical point and on the set of the preimages of the critical point i.e. satisfying following conditions: there exist $K_{E}>0$ and $\lambda_{E}>1$ such that for all $n$ :

$$
\begin{gathered}
\left|\frac{d}{d x}\left(f^{n}\right)(f(c))\right|>K_{E} \lambda_{E}^{n} \quad \text { and } \\
\left|\frac{d}{d x}\left(f^{n}\right)(z)\right|>K_{E} \lambda_{E}^{n} \quad \text { if } f^{n}(z)=c,
\end{gathered}
$$

where $c$ is the (unique) critical point of $f$ and $f^{n}=f \circ f \circ \cdots \circ f n$-times. Collet and Eckmann conjectured that $\mathbb{C} 1$ implies $\mathbb{C} 2$. This has been proven [N1] for symmetric mappings (that means such that $f(x)=f(1-x)$ for $x \in[0,1]$ ). In the present paper we prove that the conjecture is also true for nonsymmetric S-unimodal mappings.

The condition $\mathbb{C} 1$ is fulfilled quite often [CE3], but it seems that it is not necessary $[\mathbf{B}, \mathbf{C}]$. 


\section{Notation and technical lemmas}

From now on we shall assume that the mapping $f \cdot[0 ; 1] \rightarrow[0 ; 1]$ satisfies the following conditions:

A0: $f \in C^{3}[0 ; 1] ; f(0)=f(1)=0$.

A1: $f$ is $S$-unimodal; that means that there exists a unique $c \in(0 ; 1)$ such that $f$ is increasing on $(0 ; c)$ and decreasing on $(c ; 1)$ and $S f<0$ where $S f=$ $f^{\prime \prime \prime} / f^{\prime}-3\left(f^{\prime \prime} / f^{\prime}\right)^{2} / 2$.

A2: $f^{\prime \prime}(c) \neq 0$.

We shall use the following notation: $f^{1}=f$ and for $n \geq 1 f^{n+1}=f^{n} \circ f ; x_{n}=f^{n}(x)$; $D f^{n}=d f^{n} / d x$.

Now we quote without proof some lemmas:

LEMMA 1. [CE2, II.4]. If $S f \leq 0$ then $S f^{n} \leq 0 . S f \leq 0$ implies that $\left|f^{\prime}\right|$ has no positive local minima.

LemmA 2. [CE2, II.4]. If $f$ satisfies $A 1$ and $\mathbb{C} 1$ then $\left.f\right|_{\left(c_{2}, c_{1}\right)}$ has no sinks and no attractive periodic orbits.

Remark 3. By Lemma 2 we may assume later on that $f^{\prime}(0)>1$ and that there is no other fixed point of $f$ on $(0 ; c)$.

Lemma 4. [CE1, 2.2]. There are two constants $M>0, m>0$, such that for $x \in[0 ; 1]$

$$
m|x-c| \leq\left|f^{\prime}(x)\right| \leq M|x-c|
$$

and

$$
m|x-c|^{2} / 2 \leq|f(x)-f(c)| \leq M|x-c|^{2} / 2 .
$$

We define $\hat{x}(x)$ by: $\hat{c}=c$, and for $x \neq c, f(\hat{x})=f(x)$ and $\hat{x} \neq x$.

LeMma 5. $[\mathrm{N} 2,3.4]$. For $x \neq c,|\hat{x}-c| /|x-c| \leq(M / m)^{1 / 2}$ and $\left|f^{\prime}(\hat{x}) /\right| f^{\prime}(x) \mid \leq$ $(M / m)^{3 / 2}$.

LEMMA 6. [CE1, 2.6]. If $S f<0$ and $\left.D f^{n}\right|_{(x, y)} \neq 0$ then $\left|x_{n}-y_{n}\right| \geq$ $\left(D f^{n}(x) \cdot D f^{n}(y)\right)^{1 / 2}|x-y|$.

LEMMA 7. [N1; 7]. Suppose $g \in C^{3}[u, v] ; S g \leq 0 ; g^{\prime}(u)=g^{\prime}(v)=0 ;\left.g^{\prime}\right|_{(u ; v)} \neq 0$. For $a$ fixed $x \in(u, v)$ define a function $h(t), t \in[u, v]$ by $h(t)=(g(t)-g(x)) /(t-x)$ if $t \neq x$ and $h(x)=g^{\prime}(x)$. Then $h(t)$ has only one local extremum in $(u, v)$ and it is a maximum. In particular for $a \leq t \leq b$ we have $|h(t)| \geq \min (|h(a)| ;|h(b)|)$.

LEMMA 8. [CE2, 1I.4]. The set $\mathbb{C}_{-\infty}=\bigcup_{j \geq 0} f^{-j}(c)$ is dense in $[0 ; 1]$.

\section{Preliminary estimations}

Let us denote by $\Delta_{n}$ the family of the intervals of monotonicity of $f^{n}$ i.e. $(\alpha, \gamma) \in \Delta_{n}$ if and only if $\alpha, \gamma \in \bigcup_{0 \leq j<n} f^{-j}(c) \cup\{0 ; 1\}$ and $\left.D f^{n}\right|_{(\alpha, \gamma)} \neq 0$. Let $|A|$ denote the Lebesgue measure of the set $A \subset[0 ; 1]$.

LEMMA 9. Let $\alpha^{j}$ be a sequence of preimages of $c$ such that $\left(0, \alpha^{j}\right) \in \Delta_{j}$. Then there exist two constants $K_{0}>0$ and $\lambda_{0}>1$ such that:

$$
P(j)=\min \left(\left|f^{j}\left(0, \alpha^{j+1}\right)\right| /\left|\left(0, \alpha^{j}\right)\right| ;\left|f^{j}\left(\alpha^{j+1}, \alpha^{j}\right)\right| /\left|\left(\alpha^{j+1}, \alpha^{j}\right)\right|\right)
$$


Proof. First observe that $\alpha^{0}=c$ and $\alpha^{j+1}=f^{-1}\left(\alpha^{j}\right) \cap\left(0, \alpha^{j}\right)$; so such a sequence exists. We have moreover $f^{j}\left(\alpha^{j+1}\right)=c$. By density of $\mathbb{C}_{-\infty}$, there is a $j_{0}$ such that for $j>j_{0}: \min \left(c_{1}: c_{1}-c\right)<\lambda_{0} \alpha^{j}$ where $\lambda_{0}=\left(f^{\prime}(0)\right)^{1 / 2}>1$. Hence for $j \geq j_{0}$

$$
P(j) \geq \min \left(\frac{c}{\alpha^{j+1}} ; \frac{c_{1}-c}{\alpha^{j}}\right)>1 .
$$

By Lemma 7. $D f^{j}\left(\alpha^{j+1}\right) \geq P(j)>1$, and by Lemma 6

$$
\frac{c}{\alpha^{j+1}}=\frac{f^{j}\left(\alpha^{j+1}\right)-f^{j}(0)}{\alpha^{i+1}-0} \geq\left(D f^{j}(0) \cdot D f^{j}\left(\alpha^{j+1}\right)\right)^{1 / 2}>\lambda_{0}^{j} .
$$

Therefore $\alpha^{j}<\left(c / \lambda_{0}\right) \lambda_{0}^{-j}$ for all $j>j_{0}$ and

$$
P(j)>\frac{\min \left(c ; c_{1}-c\right)}{\lambda_{0}} \cdot \lambda_{0}^{j} \text { for } j>j_{0} .
$$

We set $K_{0}=\lambda_{0}^{-j_{0}} \min _{j \leq j_{0}}\left(P(j) ; c ; c_{1}-c\right)$ and obtain the assertion.

COROLLARY 10. There is a constant $K_{1}$ and a sequence $\beta^{j}$ such that for all $j\left(\beta^{j} ; 1\right) \in \Delta_{j}$ and

$$
\min \left(\left|f^{j}\left(\beta^{j+1}, 1\right)\right| /\left|\left(\beta^{j+1} ; 1\right)\right| ;\left|f^{j}\left(\beta^{j} ; \beta^{j+1}\right)\right| /\left|\left(\beta^{j} ; \beta^{j+1}\right)\right|\right)>K_{1} \lambda_{0}^{j} .
$$

Proof. We set $\beta^{j}=\hat{\alpha}^{j}$, use Lemma 9 and Lemma 5. $K_{1}=K_{0} \cdot(m / M)^{1 / 2}$.

We denote by $(a, b)$ the interval with endpoints $a, b$ independing on their mutual order (i.e. not necessary $a<b$ ).

LemMA $11[\mathrm{~N} 2,10]$. Let $(a, c) \in \Delta_{n+1} \backslash \Delta_{n}$, then there are two periodic points of period $n, p$ and $q$ such that:

$$
(q, \hat{q}) \subset(a, \hat{a}) \subset(\hat{p}, p) \text { and }\left.D f^{n}\right|_{(c, p)} \neq 0 .
$$

Moreover $a_{n}=\hat{a}_{n}=c$.

Proposition 12. Suppose that $f$ satisfies $\mathrm{A} 0-\mathrm{A} 2$ and $\mathbb{C} 1$. Then there are two constants $K_{A}>0$ and $\lambda_{A}>1$ (independent on $n$ ) such that if $(a, c) \in \Delta_{n+1} \backslash \Delta_{n}$ then

$$
\left|f^{n}(a, c)\right| /|(a, c)|>K_{A} \lambda_{A}^{n} \quad n=1,2, \ldots
$$

Proof. Assume first that $f^{n}$ is decreasing on $(c, a)$. By Lemma $11(c, q) \subset(c, a) \subset(c, \hat{p})$ for some periodic points $q=q_{n}$ and $p=p_{n}$. We study $f^{n}$ on the interval $\left(a_{1}, c_{1}\right)$. We have $q_{1} \in\left(a_{1} ; c_{1}\right)$ and $f^{n}\left(q_{1}\right)=q_{1}$, hence by Lemma 2, $\left|D f^{n}\left(q_{1}\right)\right|>1$. By Lemma 6, $\mathbb{C} 1$ and Lemma 4 we have:

$$
\begin{aligned}
\left|q_{n+1}-c_{n+1}\right| & \geq\left(D f^{n}\left(q_{1}\right) D f^{n}\left(c_{1}\right)\right)^{1 / 2}\left|q_{1}-c_{1}\right| \geq\left(K_{E} \lambda_{E}^{n}\right)^{1 / 2}\left|q_{1}-c_{1}\right| \\
& \geq\left(K_{E} \lambda_{E}^{n}\right)^{1 / 2} \frac{m}{2}|q-c|^{2} .
\end{aligned}
$$

Now we come back to the interval $(c, a)$ and as $f$ has no attractive periodic orbit we have by Lemma 5 and Lemma 1

$$
\left|D f^{n}(x)\right| \geq \min \left(\left|D f^{n}(q)\right| ;\left|D f^{n}(p)\right|\right) \geq(m / M)^{3 / 2}
$$

for all $x \in(q, p)$. We used

$$
\begin{aligned}
D f^{n}(\hat{p}) & =D f^{n-1}\left(p_{1}\right) \cdot f^{\prime}(\hat{p})=D f^{n-1}\left(p_{1}\right) \cdot f^{\prime}(p) \cdot \frac{f^{\prime}(\hat{p})}{f^{\prime}(p)} \\
& \geq D f^{n}(p)\left(\frac{m}{M}\right)^{3 / 2} .
\end{aligned}
$$


Thus

$$
|c-q| /|a-q|=\left|a_{n}-q_{n}\right| /|a-q| \geq(m / M)^{3 / 2}
$$

and

$$
|a-c|=|c-q|+|a-q| \leq|c-q|\left(1+(M / m)^{3 / 2}\right) .
$$

By (*), Lemma 4 and the last inequality

$$
\begin{aligned}
M\left|c_{n}-a_{n}\right|^{2} / 2 & \geq\left|c_{n+1}-a_{n+1}\right|>\left|c_{n+1}-q_{n+1}\right|>\left(K_{E} \lambda_{E}^{n}\right)^{1 / 2} m|q-c|^{2} / 2 \\
& \geq\left(K_{E} \lambda_{E}^{n}\right)^{1 / 2} m\left(m^{3 / 2} /\left(M^{3 / 2}+m^{3 / 2}\right)\right)^{2}|a-c|^{2} / 2
\end{aligned}
$$

Thus $\left|c_{n}-a_{n}\right| /|c-a|>K_{B} \lambda_{A}^{n}$ where $\lambda_{A}=\lambda_{E}^{1 / 4}$ and

$$
K_{B}=K_{E}^{1 / 2} m^{2} /\left(M^{2}+m M \sqrt{m}\right) .
$$

Now consider that $f^{n}$ is increasing on $(a, c)$. Then it is decreasing on $(c, \hat{a})$ and by previous estimations and Lemma 5 we have:

$$
\frac{\mid f^{n}(c)-f^{n}(a)}{|c-a|}=\frac{\left|f^{n}(c)-f^{n}(\hat{a})\right|}{|c-\hat{a}|} \cdot \frac{|c-\hat{a}|}{|c-a|} \geq K_{B} \lambda_{A}^{n}(m / M)^{1 / 2} .
$$

The assertion of Proposition 12 follows for $K_{A}=K_{B}(m / M)^{1 / 2}$.

Remark 13. By Proposition 12 there is an $N_{0}$ such that for $n>N_{0}$ if $(c, a) \in \Delta_{n+1} \backslash \Delta_{n}$ then $\left|c_{n}-a_{n}\right| /|c-a|>\lambda_{A}^{n / 2}$.

\section{The intervals of monotonicity}

The intention of the following estimations is as follows: we try to generalise Proposition 12 on all intervals $(\alpha, \beta) \in \Delta_{n+1} \backslash \Delta_{n}$. For this we assume without loss of generality that for $(\alpha, \beta) \in \Delta_{n+1} \backslash \Delta_{n}$ there is a $k<n$ such that $\alpha_{k}=\beta_{n}=c$. We write

$$
\frac{\left|\alpha_{n}-\beta_{n}\right|}{|\alpha-\beta|}=\frac{\left|\alpha_{n}-\beta_{n}\right|}{\left|\alpha_{k}-\beta_{k}\right|} \frac{\left|\alpha_{k}-\beta_{k}\right|}{|\alpha-\beta|},
$$

$\left(\alpha_{k}, \beta_{k}\right)=\left(c, \beta_{k}\right) \in \Delta_{n-k+1} \backslash \Delta_{n-k}$ and the first quotient may be estimated by Proposition 12. As for the second we observe that for some $(\gamma, \delta) \in \Delta_{k},(\alpha, \beta) \subset(\gamma, \delta)$ and both $(\gamma, \alpha)$ and $(\alpha, \delta)$ belong to $\Delta_{k+1} \backslash \Delta_{k}$. We can use Lemma 7 with $g=f^{k}$ and

$$
x=\frac{\left|\alpha_{k}-\beta_{k}\right|}{|\alpha-\beta|} \geq \min \left(\frac{\left|\alpha_{k}-\gamma_{k}\right|}{|\alpha-\gamma|} ; \frac{\left|\alpha_{k}-\delta_{k}\right|}{|\alpha-\delta|}\right)
$$

and we reduced the estimation for an interval from $\Delta_{n+1} \backslash \Delta_{n}$ to the estimation for an interval from $\Delta_{k+1} \backslash \Delta_{k}, k<n$. So we could try the inductive proof. That is how the proof goes in the symmetric case, where we can set $K_{A}=1$ in homolog of Proposition 12. But in general $K_{A}<1$ and the constant may reappear and spoil the exponential growth every time we use Proposition 12 . So we are obliged to study different cases as for example $n-k \geq N_{0}$, when we can use Remark 13, change the constant $\lambda_{A}$ or find some other way to estimate $\left|\alpha_{n}-\beta_{n}\right| /|\alpha-\beta|$. In fact although we shall use the above idea we shall prove that $|\alpha-\beta|<K \lambda^{-n}(K>0, \lambda>1)$, and then conclude (Proposition 19) that $f$ has uniform hyperbolic structure on the set of periodic points (i.e. there is a $\lambda>1$ such that for all $s$, if $p_{s}=p$ then $\left|D f^{s}(p)\right|>\lambda^{s}$ ). This will enable us to use Proposition 3.9 [N2] which states that $f$ has uniform hyperbolic structure on the set of periodic points if and only if it satisfies $\mathbb{C} 2$. 
Before we generalise Proposition 12 we introduce some new object, which will describe precisely the intervals $(\alpha, \beta) \in \Delta_{n+1} \backslash \Delta_{n}$.

For an $x \in(0 ; 1) \backslash \mathbb{C}_{-\infty}$ we define the family $\xi(x)$ of the intervals by:

$$
\xi(x)=\left\{\xi \in \bigcup_{n=0}^{\infty} \Delta_{n}: x \in \xi\right\} .
$$

Since for $k<n$ the critical points of $f^{k}$ are also the critical points for $f^{n}$ (this follows from $\left.D f^{n}(y)=D f^{n-k}\left(y_{k}\right) \cdot D f^{k}(y)\right)$ we have a natural order in $\xi(x)$ by inclusion. We numerate the intervals $\xi \in \xi(x)$ accordingly $\xi_{0}=(0 ; 1)$ and $\xi_{i+1} \subsetneq \xi_{i}$, $i=0,1, \ldots$

By density of $\mathbb{C}_{-\infty}$ we have two easy lemmas.

\section{LEMMA 14.}

(A) $\bigcap_{\xi_{i} \in \xi(x)} \operatorname{cl}\left(\xi_{i}\right)=x$.

(b) There is a $i_{0}=i_{0}(x)$ such that $\{0 ; 1\} \cap \xi_{i 0}=\varnothing$.

LEMMA 15. For every $i$ there is a point $\beta^{i} \in \xi_{i}$ such that $f^{n_{i}}\left(\beta^{i}\right)=c$, where $n_{i}$ is the maximal $n$ with $\xi_{i} \in \Delta_{n}$. Moreover $\beta^{i}$ is the endpoint of $\xi_{i+1}$.

Lemma 16. Suppose $i \geq i_{0}$ and let $\xi_{i}=(\alpha, \gamma)$. Then there are $k, m<n_{i}$ such that $\alpha_{k}=\gamma_{m}=c, k \neq m$. If $m<k$ then $k=n_{i-1}$ and $m=n_{i-l}$, for some $l \geq 2$.

Proof. As $i \geq i_{0}$ and $\xi_{i} \in \bigcup_{n=0}^{\infty} \Delta_{n}$ the endpoints of $\xi_{i}$ belong to $\mathbb{C}_{-\infty}$. As $\xi_{i} \in \Delta_{n_{i}}$ we have $k, m<n_{i}, k \neq m$ by Rolle's theorem. We have $\xi_{i} \subset \xi_{i-1} \subset \xi_{i-2}$ and it is enough to remark that $\alpha=\beta^{i-1},\left(\beta^{i}\right.$ defined in Lemma 15).

Let $\xi_{i}=\left(\alpha_{i} \gamma\right)$ and $\beta=\beta^{i} \in \xi_{i}$. We shall denote $(\alpha, \beta)$ by $\bar{\xi}_{i}$ and $(\beta, \gamma)$ by $\bar{\xi}_{i}$, $\left(\alpha_{k}=\gamma_{m}=c ; k>m\right)$.

Proposition 17. Suppose $f$ satisfies A0-A2 and that the assertion of Proposition 12 is true. Then there exist positive constants $K_{T}, d, w, \lambda_{T}$ (with $\lambda_{T}>1$ ) (independent on $n$ and $x)$ such that for every $x \in(0 ; 1) \backslash C_{-\infty}$ there is a nondecreasing sequence $\left\{s_{j}(x)\right\}$ such that for all $i$ and all $\xi_{i} \in \xi(x)$ we have

$$
A(i): \quad\left|f^{n_{i}}\left(\bar{\xi}_{i}\right)\right| /\left|\bar{\xi}_{i}\right| \geq K_{T} d^{s_{i}} \lambda_{T}^{n_{i}} \text { and }\left|f^{n_{i}}\left(\bar{\xi}_{i}\right)\right| /\left|\bar{\xi}_{i}\right|>K_{T} d^{s_{i}} \lambda_{T}^{n_{i}}
$$

and

$$
B(i): \quad\left|\bar{\xi}_{i}\right| \leq(w /(1+w))^{s_{i}} \text { and }\left|\bar{\xi}_{i}\right| \leq(w /(1+w))^{s_{i}} .
$$

The idea of the proof. The proof will consist in estimating $A(i)$ with the aid of the decomposition described at the beginning of this section. We shall fix $x$ and define $s_{i}$ in such a way that $B(i)$ holds. We shall investigate several cases, in most of them $s_{i+1}=s_{i}$, and rarely $s_{i+1}=s_{i}+1$. The sequence $s_{i}$ counts how many times we are obliged to reintroduce the constant $d$ in $A(i)$. But every time we spoil $A(i)$ we diminish the length of $\xi_{i}$ by a constant factor. In fact, as we shall see in Proposition $18 A(i)$ and $B(i)$ together prove that $\left|\xi_{i}\right|$ diminish exponentially.

Proof. First we define the constant mentioned in Proposition 17. We recall that $\lambda_{A}, K_{A}$ come from Proposition $12, N_{0}$ from Remark 13, $K_{1}, \lambda_{0}$ from Corollary 10. 
We may assume $K_{A}<1$. We fix $N_{1}>N_{0}$ such that $\lambda_{A}^{N_{1} / 2}>2 \lambda_{A}^{N_{1} / 4} / K_{A}$. Let $d=$ $\min _{x \neq y}|x-y|$, where

$$
x, y \in \bigcup_{s=0}^{2 N_{1}} f^{-s}(c) \cup\left\{c_{s}\right\}_{s=0}^{N_{1}},
$$

$d>0$ otherwise $f$ would have a sink. Obviously $d<1$.

$$
\begin{aligned}
w & =\left(K_{A} d^{3}\right)^{-1}>1, \\
\lambda_{T} & =\min \left(\lambda_{A}^{1 / 4} ; \lambda_{0} ;\left(\frac{1+w}{w}\right)^{1 / 2 N_{1}}\right)>1, \\
K_{T} & =\min \left(K_{A} ; K_{1}\right)>0 .
\end{aligned}
$$

Now we fix $x \in(0,1) \backslash \mathbb{C}_{-\infty}$ and prove $A(i)$ and $B(i)$ by induction. For $j=1, n_{j}=1$. We set $s_{1}=0$ and $A(1)$ is true by Lemma 9 and Proposition 12. $B(1)$ is obvious.

Suppose that $A(j)$ and $B(j)$ are true for all $j \leq i$. We want to prove them for $j=i+1$. If $\xi_{i+1} \cap\{0,1\} \neq \varnothing$ then $i+1<i_{0}(x)$ ( $i_{0}$ defined in Lemma 14) and we use directly Corollary 10. Otherwise both ends of $\xi_{i+1}$ are critical points of $f^{n_{i+1}}$ and we have by Lemma 16: $\beta=\beta^{i+1} \in \xi_{i+1}=(\alpha, \gamma)$ with $\alpha_{n_{i}}=\gamma_{n_{i-l+1}}=\beta_{n_{i+1}}=c$. We denote $n_{i+1}=n ; n_{i}=k ; n_{i-l+1}=m ; n>k>m$. By convention $\bar{\xi}_{i+1}=(\alpha, \beta) ; \bar{\xi}_{i+1}=(\beta, \gamma)$. We have also

$$
\begin{gathered}
\left(\alpha_{k} ; \beta_{k}\right)=\left(c, \beta_{k}\right) \in \Delta_{n-k+1} \backslash \Delta_{n-k}, \\
\left(\gamma_{m}, \beta_{m}\right)=\left(c, \beta_{m}\right) \in \Delta_{n-m+1} \backslash \Delta_{n-m}, \\
\left(\gamma_{m}, \alpha_{m}\right)=\left(c, \alpha_{m}\right) \in \Delta_{k-m+1} \backslash \Delta_{k-m},
\end{gathered}
$$

and for these intervals we can use Proposition 12 and Remark 13. We shall consider three general cases

(1) $n-k>N_{1}$,

(2.1) $n-k \leq N_{1}$ and $k-m>N_{1}$,

(2.2) $n-k \leq N_{1}$ and $k-m \leq N_{1}$.

We shall divide (2.1) into four subcases and (2.2) into three subcases.

(1) $n-k>N_{1}$. We set $s_{i+1}=s_{i}$. We estimate $A(i+1)$ for $\bar{\xi}_{i+1}$.

$$
\begin{aligned}
\frac{\left|f^{n_{i+1}}\left(\bar{\xi}_{i+1}\right)\right|}{\left|\bar{\xi}_{i+1}\right|} & =\frac{\left|\alpha_{n}-\beta_{n}\right|}{|\alpha-\beta|}=\frac{\left|\alpha_{n}-\beta_{n}\right|}{\alpha_{k}-\beta_{k} \mid} \cdot \frac{\left|\alpha_{k}-\beta_{k}\right|}{|\alpha-\beta|} \\
& \geq \lambda_{A}^{(n-k) / 4} \cdot K_{T} d^{s_{i}} \lambda_{T}^{k}>K_{T} d^{s_{i+1}} \lambda_{T}^{n}
\end{aligned}
$$

For the first quotient we used Remark 13 and (1) $\left(N_{1}>N_{0}\right)$ then the definition of $\lambda_{T},\left(\lambda_{T} \leq \lambda_{A}^{1 / 4}\right)$. For the second we used $A(i)$ and Lemma 7 , as announced at the beginning of this section. The estimation of $A(i+1)$ for $\bar{\xi}_{i+1}$ is similar as $n-m>$ $n-k>N_{1}$ and $S_{i} \geq S_{i-1} . B(i+1)$ follows from the fact that

$$
|\alpha-\gamma| \geq \max (|\alpha-\beta| ;|\beta-\gamma|)=\max \left(\left|\bar{\xi}_{i+1}\right|\left|\bar{\xi}_{i+1}\right|\right)
$$

and $(\alpha ; \gamma)$ is either $\bar{\xi}_{i}$ or $\bar{\xi}_{i}$ by Lemma 16 .

So we can consider the following case:

(2.1) We assume $n-k \leq N_{1}$ and $k-m>N_{1}$.

First we remark that as in (1) since $n-m>k-m>N_{1}$ we have

$$
\left|f^{n_{i+1}}\left(\bar{\xi}_{i+1}\right)\right| /\left|\bar{\xi}_{i+1}\right| \geq K_{r} d^{s_{i}} \lambda_{T}^{n_{i}}
$$


so we have to estimate $A(i+1)$ only for $\bar{\xi}_{i+1}$. But now we cannot use Remark 13 and we shall get rid of the constant $K_{A}$ from Proposition 12 with the "help" of the constant $w$. We distinguish four subcases:

(2.1.1) $|\alpha-\beta|>w|\gamma-\beta|$ and $\left|\gamma_{k}-\beta_{k}\right|>d\left|\alpha_{k}-\beta_{k}\right|$,

(2.1.2) $|\alpha-\beta|>w|\gamma-\beta|$ and $\left|\gamma_{k}-\beta_{k}\right| \leq d\left|\alpha_{k}-\beta_{k}\right|$,

(2.1.3) $|\alpha-\beta| \leq w|\gamma-\beta|$ and $|\gamma-\beta|>w|\alpha-\beta|$,

(2.1.4) $|\alpha-\beta| \leq w|\alpha-\beta|$ and $|\gamma-\beta| \leq w|\alpha-\beta|$,

(2.1.1): we set $s_{i+1}=s_{i}$. We define $y \in(\beta ; \gamma)$ by $\left|D f^{k}(y)\right|=\left|\gamma_{k}-\beta_{k}\right| /|\gamma-\beta|$. As $n-k \leq N_{1}$ we have $\left|\alpha_{k}-\beta_{k}\right| \geq d$, and

$$
\begin{aligned}
\frac{\left|\alpha_{k}-\beta_{k}\right|}{|\alpha-\beta|} & =\frac{\left|\alpha_{k}-y_{k}\right|}{|\alpha-y|} \cdot \frac{|\alpha-y|}{|\alpha-\beta|} \cdot \frac{\left|\alpha_{k}-\beta_{k}\right|}{\left|\alpha_{k}-\beta_{k}\right|} \\
& \geq\left(D f^{k}(y) \cdot D f^{k}(\alpha)\right)^{1 / 2} \cdot \frac{d}{1} \cdot 1 \\
& =\left(\frac{\left|\gamma_{k}-\beta_{k}\right|}{|\gamma-\beta|} \cdot\left|D f^{k}(\alpha)\right|\right)^{1 / 2} d \\
& =\left(\frac{\left|\gamma_{k}-\beta_{k}\right|}{\left|\alpha_{k}-\beta_{k}\right|} \cdot \frac{\left|\alpha_{k}-\beta_{k}\right|}{|\alpha-\beta|} \cdot \frac{|\alpha-\beta|}{|\gamma-\beta|}\left|D f^{k}(\alpha)\right|\right)^{1 / 2} d \\
& \geq\left(d \cdot \frac{\left|\alpha_{k}-\beta_{k}\right|}{|\alpha-\beta|} \cdot w \cdot\left|D f^{k}(\alpha)\right|\right)^{1 / 2} \cdot d .
\end{aligned}
$$

We used Lemma 6, the assumptions of (2.1.1) and the definition of $d$. This gives by definition of $w$, Lemma 7 and $A(i)$

$$
\frac{\left|\alpha_{k}-\beta_{k}\right|}{|\alpha-\beta|} \geq d^{3} w\left|D f^{k}(\alpha)\right| \geq d^{3}\left(K_{\mathrm{A}} d^{3}\right)^{-1} K_{T} d^{s_{i}} \lambda_{T}^{k}=K_{T} d^{s_{i}} \lambda_{T}^{k} / K_{\mathrm{A}}
$$

In order to end the estimation of $A(i+1)$ for $\bar{\xi}_{i+1}$ we observe that $\left|\alpha_{n}-\beta_{n}\right| /\left|\alpha_{k}-\beta_{k}\right| \geq$ $K_{A} \lambda_{T}^{n-k}$ by Proposition 12 and that $K_{A}$ will be simplified in the product of two quotients. $B(i+1)$ is obvious as in (1).

(2.1.2): we assume $n-k \leq N_{1}, k-m>N_{1},|\alpha-\beta|>w|\alpha-\beta|$ and $\left|\gamma_{k}-\beta_{k}\right| \leq$ $d\left|\alpha_{k}-\beta_{k}\right|$. We set $S_{i+1}=S_{i} . B(i+1)$ is obvious by $B(i)$ and $A(i+1)$ for $\overline{\bar{\xi}}_{i+1}$ is already done. We estimate $A(i+1)$ for $\bar{\xi}_{i+1}$. Again we estimate only $\left|\alpha_{k}-\beta_{k}\right| /|\alpha-\beta|$ and then we use Proposition 12.

$$
\begin{aligned}
& \frac{\left|\alpha_{k}-\beta_{k}\right|}{|\alpha-\beta|}=\frac{\left|\gamma_{k}-\alpha_{k}\right|}{|\gamma-\alpha|} \cdot \frac{\left|\alpha_{k}-\beta_{k}\right|}{\left|\gamma_{k}-\alpha_{k}\right|} \cdot \frac{|\gamma-\alpha|}{|\alpha-\beta|}=\frac{\left|\gamma_{k}-\alpha_{k}\right|}{\left|\gamma_{m}-\alpha_{m}\right|} \frac{\left|\gamma_{m}-\alpha_{m}\right|}{|\gamma-\alpha|}, \\
& \frac{\left|\alpha_{k}-\beta_{k}\right|}{\left|\alpha_{k}-\beta_{k}\right|+\left|\gamma_{k}-\beta_{k}\right|} \cdot \frac{|\gamma-\alpha|}{|\alpha-\beta|} \geq\left(\lambda_{A}^{1 / 2}\right)^{k-m} \cdot\left(K_{T} d^{s_{i-1}} \lambda_{T}^{m}\right) \cdot \frac{1}{1+d} \cdot 1 \\
& \geq \frac{2}{K_{A}}\left(\lambda_{A}^{1 / 4}\right)^{k-m} \cdot K_{T} d^{s_{i}} \lambda_{T}^{m} \cdot \frac{1}{2} \geq K_{T} d^{s_{i}} \lambda_{T}^{k} / K_{A} .
\end{aligned}
$$

We used the fact that $k-m>N_{1}>N_{0}$, Remark 13, the definition of $N_{1}, A(i-1)$ with Lemma 7 for the interval $|\gamma-\alpha|$ and $S_{i-1+1} \leq S_{i}$. We conclude $A(i+1)$ for $\bar{\xi}_{i+1}$ as in (2.1.1). 
(2.1.3): we assume $n-k \leq N_{1}, k-m>N_{1},|\alpha-\beta| \leq w|\gamma-\beta|$ and $|\gamma-\beta|>w|\alpha-\beta|$. We set $s_{i+1}=s_{i}$.

$$
\begin{aligned}
\frac{\left|\alpha_{k}-\beta_{k}\right|}{|\alpha-\beta|} & =\frac{\left|\alpha_{k}-\gamma_{k}\right|}{|\alpha-\gamma|} \cdot \frac{|\alpha-\gamma|}{|\alpha-\beta|} \cdot \frac{\left|\alpha_{k}-\beta_{k}\right|}{\left|\alpha_{k}-\gamma_{k}\right|} \geq K_{T} \lambda_{T}^{k} d^{s_{i}} \cdot \frac{|\alpha-\beta|+|\beta-\gamma|}{|\alpha-\beta|} \cdot \frac{d}{1} \\
& \geq K_{T} d^{s_{i}} \lambda_{T}^{K} \cdot(1+w) \cdot d \geq K_{T} d^{s_{i}} \lambda_{T}^{k} / K_{A} .
\end{aligned}
$$

We used $A(i)$ for the interval $|\alpha-\gamma|,(2.1 .3)$ and the definition of $w$ and the definition of $d$. Now we proceed as in (2.1.1) and obtain $A(i+1)$ for $\bar{\xi}_{i+1}$.

(2.1.4): we assume $n-k \leq N_{1}, k-m>N_{1},|\alpha-\beta| \leq w|\gamma-\beta|$ and $|\gamma-\beta| \leq w|\alpha-\beta|$.

For the first time we are obliged to set $s_{i+1}=s_{i}+1 . A(i+1)$ for $\bar{\xi}_{i+1}$ is obvious as now $d^{s_{i}}>d^{s_{i+1}}$ and we can use (1). We have to prove $A(i+1)$ for $\bar{\xi}_{i+1}$ and $B(i+1)$. We begin with $B(i+1)$. We have $|\alpha-\gamma|=|\alpha-\beta|+|\gamma-\beta|$ and by (2.1.4)

$$
|\alpha-\gamma| \geq\left(1+\frac{1}{w}\right) \max (|\alpha-\beta| ;|\beta-\gamma|) \text {. }
$$

Hence

$$
\max (|\alpha-\beta| ;|\beta-\gamma|) \leq \frac{w}{1+w}|\alpha-\gamma| \leq \frac{w}{1+w}\left(\frac{w}{1+w}\right)^{s_{i}}=\left(\frac{w}{1+w}\right)^{s_{i+1}},
$$

as we applied $B(i)$ to the interval $(\alpha, \gamma)$. This proves $B(i+1)$. Now for $A(i+1)$ for $\bar{\xi}_{i+1}$ we estimate directly using $(* *)$.

$$
\begin{aligned}
\frac{\left|\alpha_{n}-\beta_{n}\right|}{|\alpha-\beta|} & =\frac{|\alpha-\gamma|}{|\alpha-\beta|} \cdot \frac{\left|\alpha_{k}-\gamma_{k}\right|}{|\alpha-\gamma|} \cdot \frac{\left|\alpha_{n}-\beta_{n}\right|}{\left|\alpha_{k}-\gamma_{k}\right|} \geq \frac{w+1}{w} \cdot \frac{\left|\alpha_{k}-\gamma_{k}\right|}{|\alpha-\gamma|} \cdot \frac{d}{1} \\
& \geq \lambda_{T}^{2 N_{1}} K_{T} d^{s_{i}} \lambda_{T}^{k} d \geq K_{T} d^{s_{i+1}} \lambda_{T}^{n} .
\end{aligned}
$$

We used the definition of $\lambda_{T}, n-k \leq N_{1}, A(i)$ applied to the interval $(\alpha, \gamma)$, the definition of $d$ and the definition of $s_{i+1}$. This proves $A(i+1)$ for $\bar{\xi}_{i+1}$ and ends the case (2.1). Now we consider the case (2.2) $n-k \leq N_{1}$ and $k-m \leq N_{1}$.

(2.2.1) $n-k \leq N_{1}, k-m \leq N_{1}$ and $|\alpha-\beta|>w|\gamma-\beta|$. We set $S_{i+1}=S_{i}$. First we remark that $\beta_{k} \in f^{-(n-k)}(c)$, thus by definition of $d:\left|\gamma_{k}-\beta_{k}\right| \geq d>d\left|\alpha_{k}-\beta_{k}\right|$. Now we can prove $A(i+1)$ for $\bar{\xi}_{i+1}$ as in (2.1.1). As for $\bar{\xi}_{i+1}$ we have

$$
\begin{aligned}
\frac{\left|\gamma_{n}-\beta_{n}\right|}{|\gamma-\beta|} & =\frac{\left|\gamma_{n}-\beta_{n}\right|}{\left|\gamma_{m}-\beta_{m}\right|} \cdot \frac{\left|\gamma_{m}-\beta_{m}\right|}{\left|\gamma_{m}-\beta_{m}\right|} \cdot \frac{\left|\gamma_{k}-\beta_{k}\right|}{|\gamma-\beta|} \geq K_{A} \lambda_{A}^{n-m} \cdot \frac{d}{1} \cdot\left(\frac{\left|\alpha_{k}-\beta_{k}\right|}{|\alpha-\beta|} \cdot d \cdot w\right) \\
& \leq K_{A} \lambda_{T}^{n-m} d^{2} K_{T} d^{s_{i}} \lambda_{T}^{k}\left(K_{A} d^{3}\right)^{-1}>K_{T} d^{s_{i+1}} \lambda_{T}^{n} .
\end{aligned}
$$

We used Proposition 12, the definition of $d$, again (2.1.1) to estimate $\left|\alpha_{k}-\beta_{k}\right| /|\alpha-\beta|$ the inequalities of (2.2.1) then the definition of $\lambda_{T}$ and $w . B(i+1)$ is obvious.

(2.2.2): we assume $n-k \leq N_{1}, k-m \leq N_{1}$ and $|\alpha-\beta| \leq|\gamma-\beta| w$ and $|\gamma-\beta|>$ $w|\alpha-\beta|$. Similarly as in (2.2.1) we have $\left|\alpha_{m}-\beta_{m}\right|>d\left|\beta_{m}-\gamma_{m}\right|$. We set $s_{i+1}=s_{i}$ and prove $A(i+1)$ for $\bar{\xi}_{i+1}$ as we proved $A(i+1)$ for $\bar{\xi}_{i+1}$ in (2.1.1). The proof of $A(i+1)$ for $\bar{\xi}_{i+1}$ goes as in (2.1.3).

(2.2.3): we assume $n-k \leq N_{1}, k-m \leq N_{1}$ and $|\alpha-\beta| \leq|\gamma-\beta| w$ and $|\gamma-\beta| \leq$ $|\alpha-\beta| w$. We set for the second time $s_{i+1}=s_{i}+1$ and for both $\bar{\xi}_{i+1}$ and $\bar{\xi}_{i+1}$ we repeat the proof of (2.1.4) for $\bar{\xi}_{i+1}$. This ends the proof in the case (2.2) and hence the proof of Proposition 17. 


\section{Consequences}

Proposition 18. Under the assumptions of Proposition 17 there exists a constant $\lambda_{N}>1$ (independent of $x$ and $i$ ) such that for all $x \in(0,1) \backslash \mathbb{C}_{-\infty}$ and every $i$

$$
\text { if } \xi_{i} \in \xi(x) \text { then }\left|\xi_{i}\right|<K \lambda_{N}^{-n_{i}} \text {, }
$$

where $K>0$ is a constant independent of $i$.

Consider

$$
\frac{1}{n_{i}} \log \frac{\left|f^{n_{i}}\left(\xi_{i}\right)\right|}{\left|\xi_{i}\right|}
$$

by Proposition 17, $A(i)$ we have

$$
\frac{1}{n_{i}} \log \frac{\left|f^{n_{i}}\left(\xi_{i}\right)\right|}{\left|\xi_{i}\right|} \geq \frac{1}{n_{i}} \log \left(K_{T} d^{s_{i}} \lambda_{T}^{n_{i}}\right) .
$$

We have two possibilities: either

$$
\frac{1}{n_{i}} \log \left(K_{T} d^{s_{i}} \lambda_{T}^{n_{i}}\right) \geq\left(\log \lambda_{T}\right) / 2
$$

and in this case

$$
\left|\xi_{i}\right| \leq\left|f^{n_{i}}\left(\xi_{i}\right)\right|\left(\lambda_{T}^{1 / 2}\right)^{-n_{i}}<\left(\lambda_{T}^{1 / 2}\right)^{-n_{i}}
$$

or

$$
\frac{1}{n_{i}} \log \left(K_{T} d^{S_{i}} \lambda_{T}^{n_{i}}\right)<\left(\log \lambda_{T}\right) / 2,
$$

and then

$$
\frac{\log K_{T}}{n_{i}}+\frac{s_{i}}{n_{i}} \log d+\frac{1}{2} \log \lambda_{T}<0
$$

We take $i_{1}$ such that

$$
\frac{\log K_{T}}{n_{i}}+\frac{1}{4} \log \lambda_{T} \geq 0
$$

for $i \geq i_{1}$ and hence for $i \geq i_{1}$ we have

$$
\frac{s_{i}}{n_{i}} \log d+\frac{1}{4} \log \lambda_{T}<0 \text { and } \quad \frac{s_{i}}{n_{i}}>\kappa \stackrel{d f}{=}-\frac{\log \lambda_{T}}{4 \log d}>0 .
$$

But in this case we have by $B(i)$

$$
\left|\xi_{i}\right|=\left|\bar{\xi}_{i}\right|+\left|\xi_{i}\right| \leq 2\left(\frac{w}{1+w}\right)^{s_{i}}<2\left(\frac{w}{1+w}\right)^{\kappa n_{i}} .
$$

In order to finish the proof we set

$$
\lambda_{N}=\min \left(\lambda_{T}^{1 / 2} ;\left(\frac{1}{1+w}\right)^{-\kappa}\right) \geq 1 .
$$

The constant $K$ is adjusted according to $i_{1}$. 
LeMmA 19. Suppose that $f$ satisfies $\mathrm{A} 0-\mathrm{A} 3$ and there is $\lambda_{N}>1$ such that for all $x$ there is a constant $K(x)$ such that for all $i$ :

$$
\text { if } \xi_{i} \in \xi(x) \text { then }\left|\xi_{i}\right|<K(x) \lambda_{N}^{-n_{i}} .
$$

Then $f$ has uniform hyperbolic structure on the set of periodic points: i.e. if $p=p_{s}$ then $\left|D f^{s}(p)\right| \geq \lambda_{N}^{s}$.

Proof. Suppose that Lemma 19 is not true. Then there exists a periodic point $p$ of period $S, p_{s}=p$, such that $\left|D f^{s}(p)\right|=\lambda^{s}<\lambda_{N}^{s}$. we may assume $D f^{s}(p)>0$, otherwise we take $p$ and $2 s$. Take $\alpha, \beta \in \bigcup_{0 \leq r \leq s} f^{r}(c)$ such that $p \in(\alpha, \beta)$ and there is no smaller interval of such a property. We construct the intervals $\left(\alpha^{j} ; \beta^{j}\right)$ by $\alpha=\alpha$, $\beta^{1}=\beta$ and $\alpha^{j+1}=f^{-s}\left(\alpha^{j}\right) \cap\left(\alpha^{j} ; p\right)$ and $\beta^{j+1}=f^{-s}\left(\beta^{j}\right) \cap\left(p, \beta^{j}\right)$. By virtue of Lemmas 1,2 and $D f^{s}(p)>0$ such interval exists for every $j$. By Lemma $1 D f^{s}(p)>D f^{s}(x)$ either for all $x \in\left(\alpha^{1}, p\right)$ or for all $x \in\left(p, \beta^{1}\right)$. Suppose the first possibility holds (the other case is similar). Then for every $j D f^{j s}(p)>D f^{j s}(y)$ for all $y \in\left(\alpha^{j}, p\right)$. In order to prove it we decompose

$$
D f^{j s}(y)=\prod_{r=0}^{j-1} D f^{s}\left(y_{r s}\right)
$$

and as by construction of $\alpha^{j}$ for every $0<r<j$

$$
y_{r s} \in\left(\alpha^{j-r}, p\right) \subset\left(\alpha^{1}, p\right)
$$

we have $D f^{j s}(y)<\left(D f^{s}(p)\right)^{j}=D f^{s j}(p)$. Hence

$$
\lambda^{s j}=D f^{s j}(p)>\frac{\left|f^{s j}\left(\alpha^{j}, p\right)\right|}{\left|\left(\alpha^{j} ; p\right)\right|}=\frac{\left|f^{s}(\alpha)-p\right|}{\left|\alpha^{j}-p\right|} \text {. }
$$

Now consider $\xi(p)$. We have for all $j\left(\alpha^{j}, \beta^{j}\right) \in \xi(p)$ with $\left(\alpha^{j} ; \beta^{j}\right)=\xi_{i(j)}$ and obviously $\left|s \cdot j-\eta_{i(j)}\right| \leq s$. Hence by the assumption

$$
\lambda^{s j}>\frac{\left|f^{s}(\alpha)-p\right|}{K(p) \cdot L^{s}} \lambda_{N}^{s j}, \quad \text { where } L=\sup _{x \in(0,1)}\left|f^{\prime}(x)\right| .
$$

The factor $\left|f^{s}(\alpha)-p\right| / K(p) L^{s}$ does not depend on $j$ so the last inequality contradicts $\lambda<\lambda_{N}$. This proves Lemma 19.

Corollary 20. $f$ satisfies $\mathbb{C} 2$.

Proof. By Proposition 3.9, [N2]. For $S$-unimodal functions $\mathbb{C} 2$ is equivalent to uniform hyperbolic structure of the set of periodic points.

COROLlary 21. There exist a constant $\lambda>1$ such that for every $n$ and every $\xi \in \Delta_{n}$, $|\xi|<\lambda^{-n}$.

Proof. This follows from Proposition 5.2 [N2] which states that $\mathbb{C} 1$ and $\mathbb{C} 2$ imply uniform exponential diminution of length of the intervals of monotonicity.

COROLLARY 22. There are constants $K>0$ and $\lambda>1$ such that for every $x \in(0 ; 1) \mathbb{C}_{-\infty}$ and every $i$ if $\xi_{i} \in \xi(x)$ then $\left|f^{n_{i}}\left(\xi_{i}\right)\right| /\left|\xi_{i}\right|>K \lambda^{n_{i}}$.

Proof. One has to remake the proof of Proposition 17 and everytime when $A(i)$ has to be spoiled (i.e. $s_{i+1}=s_{i}+1$ ) by adding a factor $d$ one apply directly Corollary 21 as then $\left|f^{n_{i}}\left(\xi_{i}\right)\right| \geq d$. 


\section{Final remarks}

The last three corollaries show that there are no differences between hyperbolic properties of $S$-unimodal functions satisfying $C 1$ depending on symmetry. One would like to show this through a diffeomorphic conjugation of a unimodal function $f$ with symmetrical unimodal function $g$ (with $g=h \circ f \circ h^{-1}$, where $h(\hat{x})=$ $(1+x-x) / 2$ ), but the $S$ property may not be conserved.

Proposition 17 does not need $\mathbb{C} 1$ but only the assertion of Proposition 12, which is true for example for $f$ with uniform hyperbolic structure on periodic points and a weak assumption: $\left|D f^{k}\left(c_{1}\right)\right|>K \lambda_{N}^{-n / 2} n=1,2, \ldots[\mathrm{N} 2,3]$. It is tempting to prove Proposition 12 only with $\mathbb{C} 2$.

\section{Acknowledgements}

I would like to thank F. Ledrappier and J. M. Strelcyn for their effort in organising my journey to the Laboratoire de Probabilités of Université Paris VI where I wrote this paper. I am very indebted to E. Visinescu from the Laboratoire de Topologie of the Université de Dijon for hospitality and long and fruitful discussions.

\section{REFERENCES}

[B, C] M. Benedicks \& L. Carleson. On iterations of $1-a x^{2}$ on $(-1,1)$. Ann. Math. 122 (1985), 1-25.

[CE1] P. Collet \& J-P. Eckmann. Positive Liapunov exponents and absolute continuity. Ergod. Th. \& Dynam. Sys. 3 (1983), 13-46.

[CE2] P. Collet \& J-P. Eckmann. Iterated Maps of the Interval as Dynamical Systems Birkhaüser: Boston, Stuttgart, 1980.

[L, Y] A. Lasota \& J. A. Yorke. On the existence of invariant measures for piecewise monotonic transformations. Trans. AMS 186 (1973) 481-489.

[M] M. Misiurewicz. Absolutely continuous measures for certain maps of an interval. Publ. Math. IHES 53 (1981), 17-52.

[N1] T. Nowicki. Symmetric $S$-unimodal mappings and Liapunov exponents. Ergod. Th. \& Dynam. Sys. 5 (1985), 611-616.

[N2] T. Nowicki. On some dynamical properties of $S$-unimodal mappings. Fund. Math. 126 (1985), 27-43.

[Sz] W. Szlenk. Some dynamical properties of certain differentiable mappings. Bol. de la Soc. Mat. Mex. 24, (1979), 57-87. 